\title{
Working with schools: What employment providers need to know for successful collaboration $^{1}$
}

\author{
Teresa Grossi* and Faith Thomas \\ Indiana Institute on Disability and Community, Indiana University, Bloomington, IN, USA
}

Revised/Accepted November 2016

\begin{abstract}
.
BACKGROUND: This APSE workshop shared one state approach to interagency collaboration for transition-age youth. The purpose of the workshop was to determine value-added by working collaboratively and share preliminary results of a school-to-work project.

OBJECTIVE: Using a collaborative of employment providers embedded in schools to improve agency connections prior to leaving school and work experiences that lead to employment outcomes.

METHOD: The goal of the Collaborative was to provide employment opportunities for transition-age youth by embedding employment resources into the school to focus on agency connections, employment outcomes and reduce or eliminate duplication of services. Specifically, a provider employment specialist or Career Coach is embedded in the school to serve as a single point of contact to represent a "coalition" of providers serving on the Collaborative. The Collaborative also included Vocational Rehabilitation, schools, families and other agencies.

RESULTS: Schools with an embedded Career Coach had students connected to Vocational Rehabilitation Services and other community agencies than the comparison sites. Students had more opportunities for work experiences, internships and paid employment prior to leaving school with the employment providers continuing services after school.

CONCLUSIONS: By working together, schools and employment providers offer each other value in serving students and enhancing agency connections and employment outcomes.
\end{abstract}

Keywords: Interagency collaboration, collaborative, school-to-work, transition-to-work

\section{Introduction}

The Individuals with Disabilities Education Act (IDEA) of 2004 mandates that transition services be a coordinated set of activities that involves a resultoriented process focused on improving academic and functional achievement of students with disabilities

\footnotetext{
${ }^{1}$ This project was funded by The National Institute on Disability, Independent Living, and Rehabilitation Research Grant \#H133A130028.

*Address for correspondence: Teresa Grossi, Ph.D., Director of Strategic Developments, Indiana Institute on Disability and Community, Indiana University, 1905 N. Range Road, Bloomington, IN 47408, USA. Tel.: +1 812855 4070; Fax: +1 812855 9630; E-mail: tgrossi@indiana.edu.
}

as they move onto post-school settings. This provision implies the collaboration with and involvement of outside agencies so that students and families can successfully access services after leaving high school.

A 2008 report of a study conducted through the National Council on Disability (NCD), titled "The Rehabilitation Act: Transition Outcomes and Effects," concluded that youth with disabilities are not accessing or benefiting from adequate services, programs, and supportive interventions needed to successfully navigate the transition to employment and further education and training. Other significant limitations cited in the NCD report, as well as other sources, include the failure of various parties to: 
- understand roles and responsibilities in the transition process (Noonan et al., 2008; Plotner et al., 2012)

- enter into interagency agreements that accurately reflect these obligations (Grigal et al., 2011)

- share critical information related to the IEP/transition planning process (Finn \& Kohler, 2010); and

- accurately assess the capacity of different partners to fully deliver all promised services and interventions.

Federal legislation and policies have recently aligned in order to improve the kind of services and the ways they are delivered to people with disabilities. Examples of these legislative and policy realignments include a January 2014 requirement from the Centers for Medicare and Medicaid Services (CMS), calling on states to review and evaluate their Medicaid Home and Community-Based Services (HCBS) settings to ensure that people with disabilities are integrated in and have opportunities to seek employment, work in competitive and integrated settings, and engage in community life. Additionally, the Workforce Innovation and Opportunity Act of 2014 (WIOA) addresses an increased role for Vocational Rehabilitation Services as states provide both employment supports to adults with disabilities and services to transitioning youth.

This paper will share a collaborative transition model and the preliminary results of a 5-year study evaluating the effects of embedded employment resources in schools, the impact on agency connections, employment outcomes, and lessons learned from the field, all of which served as the basis for a workshop we conducted as part of the National APSE Conference.

\section{Guiding questions}

Three major questions guided the workshop discussions for transition specialists, employment providers, vocational rehabilitation staff, and advocates.

1. What do employment providers need to understand about the landscape of schools and secondary transition services?

2. What are the benefits for schools working with adult employment providers?
3. What are the benefits for employment providers working with schools?

\subsection{Understanding working with schools}

A key factor in working with any partner organization is understanding the culture of that organization. Working with school districts becomes more complex given that both the district and the individual school could have different cultures. Understanding the school's culture is often difficult for an employment provider staff as a "guest" who comes in for only short periods of time for meetings or brief interactions with students. The employment staff may have misunderstandings in communication, perceptions, and/or employment goals. Some important factors that influence school culture include:

- The continued priority of teaching state standards to ensure students graduate with a diploma or meet their state accountability system. The hope is that by increasing graduation rates for students with disabilities, the gap will close between students with and without disabilities (Every Student Succeeds Act, 2015). This results in more time and resources devoted to improving graduation rates and less time for career development activities and/or life skill instruction.

- Many school districts are faced with teacher shortages, few qualified applicants, and/or a lack of substitutes, especially for teaching students with higher support needs. This shortage impacts the quality of instruction and/or opportunities for teachers to engage in professional development activities and collaborative activities with other professionals.

- A high number of schools are now using technology for instruction and classroom assignments. Some districts ensure that every student has access to either a laptop or tablet. Students are leaving school with a technology-savvy skill set. Employment providers need to be prepared to continue the use of technology for everyday life, instruction, and support strategies.

- Many schools have a transition coordinator or work-study coordinator to assist with workbased learning and other transition needs. Each district may approach this position differently. For example, some transition coordinators may be more administrative versus hands-on with students. In other districts, the transition coordinator may serve only a certain group of students 
(e.g., higher support needs). Understanding the role and responsibilities of this staff person is important to enhance collaboration.

- Teachers are often asked to play a variety of roles and have expertise in a number of areas. The most important skill-set, however, is the ability to provide quality instruction, which impacts academic skills such as reading, writing, and math. Therefore, the teacher's knowledge of community resources, employment services, and employer needs are often limited.

\subsection{Benefits of employment providers working with schools}

There are a variety of reasons why employment providers should work with schools. A few include:

- Schools are a pipeline of referrals to Vocational Rehabilitation Services (VRS) and employment providers.

- Teachers know their students and families, their strengths, instructional and support strategies. They know what has worked and not worked for an individual student (e.g., accommodations, experiences, and activities).

- Schools can facilitate the relationship between the students and families with the employment provider. Teachers often build years of trust with families, who then rely on them for guidance and direction for services. Maneuvering the adult system of services is overwhelming, complex, and frustrating for many families. A seamless system of transition would allow for a period of overlap, ensuring that students and families have the safety net of schools while transitioning to services with adult providers.

- Schools have information from age-appropriate transition assessments as well as other student work experiences and activities which will help inform employment providers as they initiate the employment process with a student. This information can be critical to begin the Discovery process, create work experiences, or conduct job development.

\subsection{Benefits of schools working with employment providers}

Employment providers offer schools a number of benefits, including but not limited to:
- An age-appropriate approach with transitionage youth. Employment providers tend to view transition-age youth as young adults rather than as students.

- Knowledge and experience working with employers and the local labor market. Employment providers have ongoing relationships with area businesses, work with local Chambers of Commerce, and understand the needs of employers.

- Knowledge of other adult agencies, which means they can assist school personnel, students and families in making appropriate connections.

- Awareness of state and federal changes affecting services (e.g., Medicaid Waiver services, the referral and eligibility process, work incentives).

- The ability to supplement what schools are currently doing to enhance work-based learning experiences and facilitate connections to employers and other agencies, as needed. Some schools have limited resources and/or staff. Overwhelmed classroom teachers may default to in-school jobs or more group work experiences rather than individualized experiences. Employment providers assist and fill in the gaps, offering schools best practices in employment services.

\section{One example: Indiana School-to-Work Collaborative}

The Indiana School-to-Work Collaborative was designed by representatives of schools, employment providers, IN-APSE, Vocational Rehabilitation Services, and other advocacy stakeholders to promote a more seamless system of transition and collaboration by embedding employment resources in the school. This model was developed prior to WIOA 2014 and the HCBS Waiver rules of 2014. Unique aspects of the Collaborative include:

1. Community employment providers (community rehabilitation programs and mental health agencies) "opt-in" to be part of the School-to-Work Collaborative for their local community. Each Collaborative has key stakeholders including schools, VRS, providers, parent representatives and other local agencies.

2. A single-point-of-contact "Career Coach," employed by one of the community 
rehabilitation providers, represents the Collaborative and is embedded in the school, solely focusing on employment outcomes and access to services for students/families. By being immersed in the school culture, the Career Coach gets to know the students, provides outreach to potential VRS-eligible consumers, connects families to other agencies, partners on skill training, oversees internship or work experience sites, and provides consultation to quality work experiences that lead to an employment outcome.

3. The Discovery Profile documents the student's attributes, strengths, preferences, interests, and support needs to aid in identifying potential internship sites as well as job searches and job matches that lead to quality employment outcomes. Vocational Rehabilitation Services pays for the Discovery process.

4. Prior to their last year in high school, students participate in internships and/or paid and nonpaid work experiences.

5. Schools implement research-based selfdetermination curricula to teach students to lead and/or be more active in their Transition IEP planning process. Students participate to increase leadership skills, problem-solving, choice-making, goal setting and other skills. As a part of the experience, students interview and choose their employment provider.

6. Benefits Information Network (BIN) liaisons, funded by VRS, provide benefits planning consultation to the student and family.

7. IN*SOURCE, Indiana's parent training and information center, provides information to families about the Collaborative, training, helps them create a vision for the future, raises expectations about employment, and mentors families as needed.

\subsection{Targeted students}

Those targeted for participation in their School-toWork Collaborative were students with a disability who had difficulty meeting the diploma requirements, those who had already chosen to leave school without a diploma, those who were meeting diploma requirements but had not yet had any work experiences prior to leaving school, or those whose postsecondary goal was to enter the workforce upon leaving high school. The majority of students served in the collaborative were students with learning disabilities (31\%), intellectual disabilities (24\%), emotional disabilities (14\%) and autism (14\%)

\subsection{Preliminary outcomes and lessons learned}

Table 1 compares the connections with adult services for the Collaborative sites to the control sites, those districts that did not have employment resources embedded in the schools. As indicated in Table 1, students were more likely to be connected to Vocational Rehabilitation and employment providers in those schools where resources were embedded. Those students (9) in the research sites not connected were freshman just starting the self-determination curricula.

A number of lessons have emerged from the first two years of implementation. Although this list is not exhaustive, it has assisted districts in avoiding certain issues in year three of the project.

\subsubsection{Time to plan and organize}

The first year of implementation was focused on the logistics, roles and responsibilities and operations. Recruitment occurred and internships were initiated but at a much lower rate than the second year. As we moved into the third year, more systems were in place and organized. During the first two years, there were ninety-three internships or work experiences, averaging eleven hours per week (range of 1 to 40 hours) for an average of six weeks. Data after two years of implementation includes a $22 \%$ employment rate for students leaving school with the average wage of $\$ 8.36$ (range of $\$ 7.25$ to $\$ 12.40$ ) and the average hours worked at 25 per week (range of 5 to 40 hours). The employment providers continue to work with the students who did not leave school with a job by continuing to engage in the discovery process and job development activities.

Table 1

Connections to Adult Services: Comparison of Experimental and Control Sites After Two Years of Implementation

\begin{tabular}{lcc}
\hline Agency & $\begin{array}{c}\text { Experimental Sites } \\
\mathrm{N}=208\end{array}$ & $\begin{array}{c}\text { Control Sites } \\
\mathrm{N}=66\end{array}$ \\
\hline Vocational Rehabilitation & $59 \%$ & $11 \%$ \\
Employment Provider & $13 \%$ & 0 \\
Case Management Provider & $5 \%$ & 0 \\
Developmental Disabilities & $5 \%$ & $7 \%$ \\
Day Service Provider & $2 \%$ & 0 \\
Residential Provider & $4 \%$ & 0 \\
WorkOne & 0 & $6 \%$ \\
Juvenile Justice & $1 \%$ & $4 \%$ \\
Mental Health Center & $8 \%$ & $9 \%$ \\
Not connected to any agency & $4 \%$ & $61 \%$ \\
\hline
\end{tabular}




\subsubsection{Understanding working with youth and their culture}

For employment consultants, it was imperative to understand how to work with adolescents. We found early on that youth expected rapid engagement from the employment consultant. For example, because they were in a school-to-work program, students expected to participate on a daily or at least weekly basis. This required the employment consultant to make regularly scheduled meetings.

\subsubsection{Communication modes and audiences}

Understanding the need for multi-faceted communication became increasingly critical to ensure all players were moving in the right directions. This included the students and families, department chairs, individual teachers, and other relevant stakeholders. For students, many preferred texting, while others needed an email, phone call, or face-to-face interaction.

\subsubsection{Technology and use of apps}

Many students were accustomed to learning through tablets and apps. Collaboratives recognized the need to coordinate and carry over the use of technology to the workplace and community settings.

\subsubsection{Preparing for staff turnover}

Collaboratives found that anticipating turnovers for both teachers and agency employment consultants helped reduce the length of service interruptions.

\subsubsection{Supplementing current school activities}

The development of "onboarding" materials and documentation helped employment consultants establish a "baseline." Knowing what types of activities and materials schools were already using (e.g., age-appropriate transition assessments, transition services and activities, community-based and workbased learning experiences) helped Career Coaches identify areas where they could be of most help.

In summary, the intentions of the various legislations, policies and research is to impact students and families at the local level. We have proposed one approach to help respond to how transition professionals "scale-up" or implement best practices statewide (Fixsen et al., 2013, Test et al., 2009) and ensure that all key stakeholders involved in a student's life actually work together with a sense of urgency and not wait for "someone else do it".

\section{Conflict of interest}

The authors have no conflict of interest to report.

\section{References}

Every Student Succeed Acts (2015). Retrieved November 2, 2016 from http://www.ed.gov/essa?src=rn

Finn, J. E. \& Kohler, P. D. (2010). Transitions outcomes project: Perceptions of school personnel explored through a multiple case study. Journal of Ethnographic \& Qualitative Research, 4, 95-107.

Fixsen, D. L., Blase, K. A., Metz, A., \& Van Dyke, M. (2013). Statewide implementation of evidence-based programs. Exceptional Children (Special Issue), 79(2), 213-230.

Grigal, M., Hart, D., \& Migliore, A. (2011). Comparing the transition planning, postsecondary education, and employment outcomes of students with intellectual and other disabilities. Career Development for Exceptional Individuals, 34, 4-17.

Individuals with Disabilities Education Improvement Act of 2004, 20 U.S.C. 1400 et seq.

National Council on Disability (2008). The Rehabilitation Act: Outcomes for transition-age youth. Washington, DC.

Noonan, P. M., Morningstar, M. E., \& Erickson, A. G. (2008). Improving interagency collaboration: Effective strategies used by high-performing local districts and communities. Career Development for Exceptional Individuals, 31, 132-143.

Plotner, A., Trach, J., \& Shogren, K. (2012). Identifying a transition competency domain structure: Assisting transition planning teams to understand roles and responsibilities of community partners. Rehabilitation Research, Policy, and Education, 26, 255-270.

Test, D. W., Fowler, C. H., Richter, S. M., White, J., Mazzotti, V., Walker, A. R.,... Kortering, L. (2009). Evidence-based practices in secondary transition. Career Development for Exceptional Individuals, 32, 115-128.

U.S. Department of Health and Human Services, Centers for Medicare and Medicaid Services. Home and Community Based Services. Retrieved November 1, 2016, https:// www.medicaid.gov/medicaid/hcbs/guidance/index.html

U.S. Department of Labor, Workforce Innovation and Opportunity Act of 2014, 29 U.S.C. 